\title{
MASS TRANSFER THROUGH FREE SURFACE BOUNDARY LAYERS USING A STATISTICAL APPROACH
}

\author{
FRANCISCO ANTONIO LOYOLA LAVIN ${ }^{1} \&$ HARRY EDMAR SCHULZ ${ }^{2,3}$ \\ ${ }^{1}$ Department of Mechanical Engineering University of São Paulo, Brazil \\ ${ }^{2}$ Department of Hydraulics and Sanitary Engineering, University of São Paulo, Brazil \\ ${ }^{3}$ Department of Hydraulics and Environmental Engineering, Federal University of Ceará, Brazil
}

\begin{abstract}
Mass exchange through gas-liquid interfaces, whose liquid side has a turbulent nature, are still difficult to quantify due to the unclosed set of turbulence equations, which are also nonlinear. This paper describes an efficient method to overcome this difficulty, by substituting the statistical variables of the original equations by statistical relationships furnished by the Random Square Waves (RSW) tool. Oscillatory records are simplified using random square waves (ideal and binary), which allow a theoretical statistical treatment of the signals. This tool was applied to the concentration boundary layer at the gas-liquid interface. Normalized mass fluxes and mean concentration profiles were obtained using Taylor-series-based solutions, which allow for consideration of transient situations through the successive calculation of the higher order coefficients (derivatives). Comparisons with experimental data available in open literature are presented as a first evaluation of the Taylor series, showing promising results. This method is a viable tool, and this study shows novel conclusions that reproduce general tendencies observed in one-dimensional mass transfer phenomena in boundary layers.

Keywords: turbulent mass flux, concentration profile, gas-liquid interfaces, RSW, turbulence statistics.
\end{abstract}

\section{INTRODUCTION}

Physical phenomena that depend on gas-liquid free interfaces, such as the dissolution of substances into water bodies, still await a definitive quantification and understanding regarding the boundary layer details, mainly due the turbulent nature of the boundary layer.

In this study, the focus is the liquid side of the interface. It is known that, in addition to molecular diffusion, it is necessary to consider the mixing of the liquid promoted by the turbulent eddies created by the flow field, usually named as a turbulent diffusion [1].

Many studies correlate the so-called mass transfer coefficient and ad-hoc parameters, such as the boundary layer thickness, the mean time of exposure, or the renewal rate of the surface (see [2] and [3] for a broad review of conceptual models for interfacial transfer). A still limited number of measurements near the surface are available in the open literature [4-8], [12-14], [16], [17] conducted to confirm conclusions derived from conceptual and semiempirical models, and to shed light on the details of the turbulent movements close to mobile interfaces. However, accurate results are still difficult to obtain due the small thickness (10$100 \mu \mathrm{m}$ ) of the concentration boundary layer [8], together with optical complexities linked to the measurement technique, such as noise, surface localization, and needed corrections due to laser attenuation and to optical blurring [10], [11].

Testing possibilities of numerical tools, DNS studies were done for example by [19], [20] for isotropic turbulence and a wide range of Schmidt and Reynolds numbers. The numerical results suggest that small eddies increase their effect on the mass flux as the Reynolds and Schmidt numbers increase. It is also suggested that numerical results agree with the surface divergence model proposed by [21] and given by $K_{L} \sim \sqrt{\varphi D}$, being $K_{L}$ the mass transfer coefficient, $D$ the molecular diffusivity coefficient, and $\varphi=-\left(\frac{\partial U}{\partial x}+\frac{\partial V}{\partial y}\right)$ the surface 
divergence. $U, V$ are orthogonal superficial velocity components. It is assumed by several authors [18], [23] that $\varphi$ has a key role in transfer processes. Because divergence considers the turbulent movement of the superficial velocity components, it points to the importance of elucidating how the structure of turbulence combines with the molecular diffusion to control the mass exchange in the adjacent region of the gas-liquid interface.

In a study of long maturation, Schulz and co-workers [9], [15], [16], [25-27] progressively presented the tool "Random Square Waves", which was applied to an interface boundary layer. The starting equation is the Reynolds averaged mass conservation equation, where the Reynolds decomposition of variables, given by a mean parcel and a fluctuating parcel, is the main assumption. For the scalar field the decomposition leads to $F=\bar{F}+f$, and for the velocity field it leads to $\boldsymbol{U}=(U, V, W)=(\bar{U}+u, \bar{V}+v, \bar{W}+w)$. Eqn (1) expresses the mass conservation equation in its usual form:

$$
\frac{\partial F}{\partial t}+\boldsymbol{U} \cdot \nabla F=D \cdot \nabla^{2} F
$$

$F, \bar{F}$, and $f$ are the instantaneous, mean, and fluctuating scalar variable; $U, \bar{U}, u ; V, \bar{V}, v$; $W, \bar{W}$, and $w$ are the instantaneous, mean and fluctuating $x, y$, and $z$ components of the velocity, respectively. When generating conservation equations for the statistical moments of the scalar fluctuations (moments given by $\theta=1,2,3 \ldots$ ) eqn 2 is obtained (see [15]):

$$
\begin{gathered}
\overline{f^{\theta}} \frac{\partial \bar{F}}{\partial t}+\frac{1}{\theta+1} \frac{\partial \overline{f^{\theta+1}}}{\partial t}+\overline{f^{\theta}}[(\bar{U}, \bar{V}, \bar{W}) \cdot \nabla \bar{F}]+\frac{1}{\theta+1}\left[(\bar{U}, \bar{V}, \bar{W}) \cdot \nabla \overline{f^{\theta+1}}\right] \\
+\left(\overline{f^{\theta} u}, \overline{f^{\theta} v}, \overline{f^{\theta} w}\right) \cdot \nabla \bar{F}+\frac{1}{\theta+1} \nabla \cdot \overline{\left[(u, v, w) f^{\theta+1}\right]}=D\left[\overline{f^{\theta}} \nabla^{2} \bar{F}+\overline{f^{\theta} \nabla^{2} f}\right] .
\end{gathered}
$$

Most of the studies in the literature assume unidirectional mass flux trough a flat-interface, and no dominant velocity, so that eqn (2) can be simplified to eqn (3), taken here as the governing equation for the gas-liquid system:

$$
\overline{f^{\theta}} \frac{\partial \bar{F}}{\partial t}+\frac{1}{\theta+1} \frac{\partial \overline{f^{\theta+1}}}{\partial t}+\overline{f^{\theta} w} \frac{\partial \bar{F}}{\partial z}+\frac{1}{\theta+1} \frac{\partial \overline{w f^{\theta+1}}}{\partial z}=D\left[\overline{f^{\theta}} \frac{\partial^{2} \bar{F}}{\partial z^{2}}+\overline{f^{\theta} \frac{\partial^{2} \bar{f}}{\partial z^{2}}}\right] .
$$

According to the authors' knowledge, studies conducted until now with eqn (3) assumed the condition of uniform attenuation/reduction of the wave amplitude (scalar fluctuations) along the boundary layer. Table 1 summarizes the main studies on RSW, all for a constant value of the reduction function $\alpha_{f}$ that quantifies attenuation. Although theoretical results for concentration profiles are in good agreement with measured data, the experiments of [16] already showed that $\alpha_{f}$ is not constant. In order to obtain a better understanding of the interfacial mass exchange process, this study presents the theoretical description of the RSW method including the variable reduction function $\alpha_{f}$, and furnishes first results for transient problems with variable $\alpha_{f}$.

\section{GENERAL ASPECTS OF THE RSW METHOD}

The Random Square Waves tool approximates fluctuating records by a bimodal random function as shown in Fig. 1, with two extreme values: $F_{p}$ and $F_{n}$. Here $F_{p}$ is the saturation concentration of a soluble gas in the liquid (taken at the surface of the liquid) and $F_{n}\left(<F_{p}\right)$ is taken as the homogeneous gas concentration in the bulk liquid. 
Table 1: Chronological development of RSW method.

\begin{tabular}{|c|c|c|}
\hline Author & Regime-study type & Main results \\
\hline$[24]$ & Transient-theoretical & $\begin{array}{l}\text { Introduced RSW tool by idealizing fluctuations and } \\
\text { defining a } 1^{\text {st }} \text { statistical function (partition } n \text { ). }\end{array}$ \\
\hline $\begin{array}{l}{[9]} \\
{[16]}\end{array}$ & $\begin{array}{l}\text { Transient-theoretical } \\
\text { and experimental }\end{array}$ & $\begin{array}{l}\text { Definition of } 2^{\text {nd }} \text { statistical function (reduction } \alpha_{f} \text { ). } \\
\text { Measuring } \alpha_{f} \text { profiles in oscillating grid tank using } \\
\text { LIF. }\end{array}$ \\
\hline$[25]$ & Transient-theoretical & $\begin{array}{l}\text { Definition of } 3^{\text {rd }} \text { statistical function (superposition } \beta \text { ). } \\
\text { Concentration profile obtained numerically. }\end{array}$ \\
\hline$[15]$ & Transient-theoretical & $\begin{array}{l}\text { Concentration profile obtained for a wide range of } \alpha_{f} \\
\text { values. }\end{array}$ \\
\hline$[26]$ & Stationary-theoretical & Theoretical solutions obtained for stationary regime. \\
\hline$[27]$ & Stationary-theoretical & $\begin{array}{l}\text { Relevant functions in mass transport theoretically } \\
\text { estimated: turbulent and diffusive flux, concentration } \\
\text { central moments, velocity fluctuation RMS, among } \\
\text { others. }\end{array}$ \\
\hline
\end{tabular}

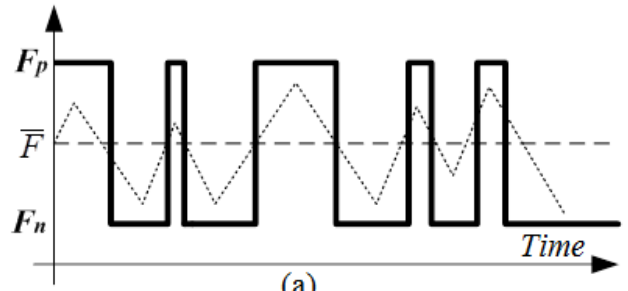

(a)

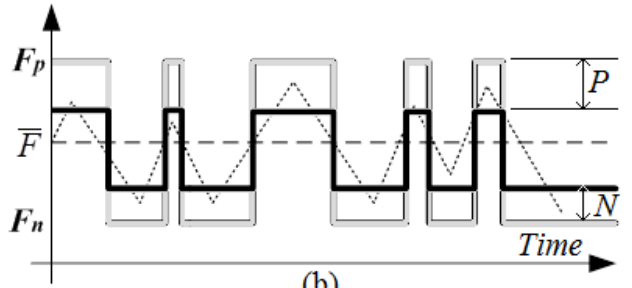

(b)

Figure 1: (a) RSW tool. Extreme values $F_{p}$ and $F_{n}$ (solid line) may not be attained by the real fluctuation (dotted line); (b) Mean amplitude reduction denoted by $P$ and $N$.

The partition function $n$ (see Table 1), ranging between 0 and 1, is the portion of time that the square wave is greater than $\bar{F}$ (or equals $F_{p}$ ). This definition implies that $n$ coincides with the normalized concentration $n=\left(\bar{F}-F_{n}\right) /\left(F_{p}-F_{n}\right)$.

The reduction function $\alpha_{f}$ (see Table 1), ranging between 0 and 1, quantifies the decay of the amplitude using the variables $P$ and $N$ of Fig. 1(b), calculated as $N=\alpha_{f} n\left(F_{p}-F_{n}\right)$ and $P=\alpha_{f}$ $(1-n)\left(F_{p}-F_{n}\right) . \alpha_{f}=0$ means no attenuation, and $\alpha_{f}=1$ means fully attenuation.

The superposition function $\beta$ (see Table 1), ranging between 0 and 1 , is the portion of time in which velocity and scalar fluctuations ( $w$ and $f$ ) are simultaneously positive.

Using $n, \alpha_{f}$ and $\beta$ in the Reynolds averaged equations, [25] presented eqn (2) for any moment $\theta$. Here eqns (4), (5), and (6) are used for $\theta=1,2$ and 3, respectively.

$$
\begin{gathered}
1-n=S \frac{d^{2} n}{d z^{* 2}}-\frac{d \Phi}{d z^{*}} \\
-n(1-n) A^{2}+\Phi \frac{d n}{d z^{*}}+\frac{1}{2} \frac{d}{d z^{*}}[\Phi A(1-2 n)]=\operatorname{Sn}(1-n) A \frac{d^{2}[(1-2 n) A]}{d z^{* 2}}
\end{gathered}
$$




$$
\begin{gathered}
n(1-n) A^{2}[-(1-2 n) A+1-n]+\Phi(1-2 n) A \frac{d n}{d z^{*}}+\frac{d}{d z^{*}} \frac{\Phi A^{2}\left(1-3 n+3 n^{2}\right)}{3} \\
=\operatorname{Sn}(1-n) A^{2}\left[\begin{array}{c}
\{1-A(1-2 n)\} \frac{d^{2} n}{d z^{* 2}}+\left\{1-2 n+2 n^{2}\right\} \frac{d^{2} A}{d z^{* 2}}+ \\
2\{2 n-1\} \frac{d n}{d z^{*}} \frac{d A}{d z^{*}}
\end{array}\right] .
\end{gathered}
$$

$\Phi$ is the dimensionless turbulent flux given by eqn (7) $\left(\Phi=I J^{*}\right.$ in the original study of [15]). $A=1-\alpha_{f}$ and $S=D / K_{L} E$ is a dimensionless diffusivity, being $E$ the length in $z^{*}$ that implies in a proper variation of $F$. $E$ is used to define the dimensionless distance $z^{*}=z / E$.

$$
\Phi=\frac{n(n-1)\left(1-\alpha_{f}\right) \frac{\sqrt{\overline{\omega^{2}}}}{E K_{L}}}{\sqrt{n(n-1)+\frac{\beta(1-\beta)}{(2 \beta-1)^{2}}}} .
$$

$\sqrt{\overline{\omega^{2}}}$ is the root mean square of the velocity fluctuations. It can be seen that the 1D RSW problem reduces to the three unknown functions $n, A$, and $\Phi$, thus needing only three equations to obtain a solution. In this sense, the RSW method leads to closed sets of equations for 1D turbulence problems.

The boundary conditions for the function $n$ are: 1) first type: $n\left(z^{*}=0.0\right)=1.0$, $n\left(z^{*} \rightarrow \infty\right)=0.0$; and 2 ) second type: $d n / d z^{*}\left(z^{*} \rightarrow \infty\right)=0.0$. In boundary layer problems the conditions imposed at $z^{*} \rightarrow \infty$ are generally not useful, so that boundary layer thicknesses are commonly defined conditionally for $n=0.01$. In the present study it was adequate to apply boundary conditions as proposed in Fig. 2.

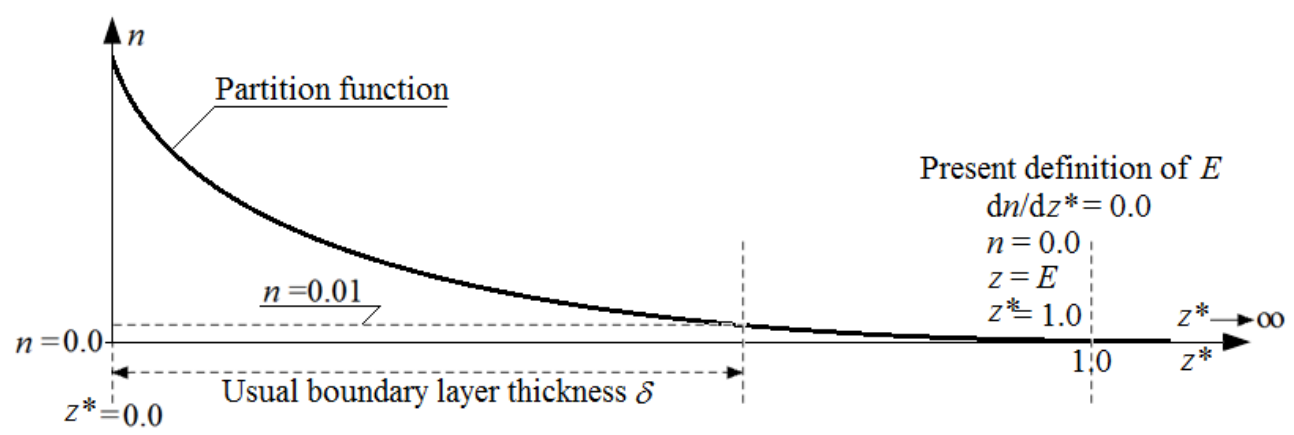

Figure 2: Boundary conditions adopted for defining $E$ in the present study, conditioned by the evolution of the partition function $n$.

The condition $d n / d z^{*}\left(z^{*}=1.0\right)=0.0$ is also shown in Fig. 2. By evaluating eqns (5) and (6) at $z^{*}=1.0$ using the shown boundary conditions, the coupled eqns (8a) and (8b) are obtained

$$
A_{z^{*}=1} \frac{d \Phi}{d z^{*}{ }_{z^{*}=1}}+\Phi_{z^{*}=1} \frac{d A}{d z^{*} z^{*}=1}=0
$$




$$
A_{z^{*}=1}\left[A_{z^{*}=1} \frac{d \Phi}{d z^{*} z^{*}=1}+2 \Phi_{z^{*}=1} \frac{d A}{d z^{*}{ }_{z^{*}=1}}\right]=0 .
$$

By assuming $A_{z^{*}=1} \neq 0.0$ and $\Phi_{z^{*}=1} \neq 0.0$, eqns (8a) and (8b) have the single solution $d \Phi / d z^{*}\left(z^{*}=1.0\right)=d A / d z^{*}\left(z^{*}=1.0\right)=0.0$. This led to take $\Phi_{z^{*}=1}$ and $A_{z^{*}=1}$ as parameters, with the simplified notation $\Phi_{1}$ and $A_{1}$. The solution $d \Phi / d z^{*}\left(z^{*}=1.0\right)=0.0$ applied into eqn (4) produces $d^{2} n / d z^{* 2}\left(z^{*}=1.0\right)=1 / S$. By firstly derivating eqns (4), (5), and (6), then evaluating them at $z^{*}=1.0$ through the know results of $d^{2} n / d z^{* 2}, d \Phi / d z^{*}$, and $d A / d z^{*}$, we obtain higher order derivatives at this position. Table 2 summarizes the derivatives of first, second, and third order.

Table 2: First, second and third order derivatives of the functions $n, A$ and $\Phi$ at $z^{*}=1.0$.

\begin{tabular}{|c|c|c|c|}
\hline \multirow{2}{*}{ Function } & \multicolumn{2}{|c|}{ Order } \\
\cline { 2 - 4 } & $1 \mathrm{st}$ & $2 \mathrm{nd}$ & $3 \mathrm{rd}$ \\
\hline$n$ & 0 & $\frac{1}{S}$ & $-\left(\frac{\Phi_{1}}{\mathrm{~A}_{1}}\right)\left(\frac{1}{S}\right)^{2}\left(1-\mathrm{A}_{1}\right)$ \\
\hline$A$ & 0 & $-\frac{\left(1-\mathrm{A}_{1}\right)}{S}$ & $-\left(\frac{\Phi_{1}}{\mathrm{~A}_{1}}\right)\left(\frac{1}{S}\right)^{2}\left(1-\mathrm{A}_{1}\right)^{2}-\left(\frac{\mathrm{A}_{1}}{\Phi_{1}}\right)\left(\frac{1}{S}\right)\left(1-3 \mathrm{~A}_{1}\right)$ \\
\hline$\Phi$ & 0 & $-\left(\frac{\Phi_{1}}{\mathrm{~A}_{1}}\right) \frac{\left(1-\mathrm{A}_{1}\right)}{S}$ & $\left(\frac{\Phi_{1}}{\mathrm{~A}_{1}}\right)^{2}\left(\frac{1}{S}\right)^{2}\left(1-\mathrm{A}_{1}\right)^{2}-\left(\frac{1}{S}\right)\left(1+3 \mathrm{~A}_{1}\right)$ \\
\hline
\end{tabular}

This inductive process can be repeated until the desired accuracy (error) of the functions $n, A$, and $\Phi$ at a desired position is attained, related to the number of terms in Taylor series of these functions. The position for the control of the quality of the obtained solution is chosen as $z^{*}=0.0$, where it is known that $n=1.0$. The power series can be expressed for $n, A$ and $\Phi$ as given in eqns (9a), (9b) and (9c), respectively:

$$
\begin{aligned}
& n=n_{z^{*}=1}+\left(z^{*}-1\right) \frac{d n}{d z^{*} z^{*}=1}+\left(z^{*}-1\right)^{2}{\frac{d^{2} n}{d z^{* 2}}}_{z^{*}=1}+\left(z^{*}-1\right)^{3} \frac{d}{3} n_{d z^{* 3}}+\cdots \\
& A=A_{z^{*}=1}+\left(z^{*}-1\right) \frac{d A}{d z^{*}{ }^{*}=1}+\left(z^{*}-1\right)^{2}{\frac{d^{2} A}{d z^{* 2}}}_{z^{*}=1}+\left(z^{*}-1\right)^{3} \frac{d^{3} A}{d z^{* 3}}{ }_{z^{*}=1}+\cdots \\
& \Phi=\Phi_{z^{*}=1}+\left(z^{*}-1\right) \frac{d \Phi}{d z^{*}{ }^{*}=1}+\left(z^{*}-1\right)^{2}{\frac{d^{2} \Phi}{d z^{* 2}}}_{z^{*}=1}+\left(z^{*}-1\right)^{3} \frac{d}{3} \Phi_{d z^{* 3}}+\cdots
\end{aligned}
$$

The first lower order derivatives of Table 2 convert eqns (9a), (9b), and (9c) into eqns (10a), (10b), and (10 c).

$$
\begin{gathered}
n=\frac{\left(z^{*}-1\right)^{2}}{S}-\left(\frac{\Phi_{1}}{A_{1}}\right) \frac{\left(1-A_{1}\right)\left(z^{*}-1\right)^{3}}{S^{2}}+\cdots \\
A=A_{1}-\frac{1-A_{1}}{S}\left(z^{*}-1\right)^{2}-\left[\left(\frac{\Phi_{1}}{A_{1}}\right)\left(\frac{1-A_{1}}{S}\right)^{2}-A_{1}\left(\frac{1-3 A_{1}}{S \Phi_{1}}\right)\right]\left(z^{*}-1\right)^{3}+\cdots,(10
\end{gathered}
$$




$$
\Phi=\Phi_{1}-\frac{\Phi_{1}\left(1-A_{1}\right)\left(z^{*}-1\right)^{2}}{S A_{1}}+\left(z^{*}-1\right)^{3}\left\{\left[\frac{\Phi_{1}\left(1-A_{1}\right)}{S A_{1}}\right]^{2}-\frac{1+3 A_{1}}{S}\right\}+\cdots
$$

To obtain higher order coefficients a code was developed in the MATLAB ${ }^{\circledR}$ symbolic environment furnishing the vector $\left[n^{(p)}, A^{(p)}, \Phi^{(p)}\right]$, where $(p)$ indicates de $p^{\text {th }}$ order derivative. Eqns (10a), (10b), and (10c) also show that higher values of $S$ imply in a lower number of terms in the three power series. This procedure (higher value of $S$ ) is used here to check the quality of the profiles obtained and the needed convergence.

Further, the experimental trend obtained by [16] for $\alpha_{f}$ was used as a boundary condition for $A=1-\alpha_{f}$ in the form $A\left(z^{*}=0\right)=0$, while the conclusions of the experimental and theoretical analysis of [11] were used as boundary conditions for $\Phi$ in the form $\Phi\left(z^{*}=0\right)=0$ and $d \Phi / d z^{*}\left(z^{*}=0\right)=0$. This implied adding a 19th parcel to the Taylor series for $n, A$, and $\Phi$, and also a 20th parcel to the Taylor series of $\Phi$.

\section{OBTAINED PROFILES AND QUALITATIVE COMPARISON WITH EXPERIMENTAL DATA}

Fig. 3 depicts the theoretical profiles of $n, A$, and $\Phi$ for $0.0 \leq z / \delta \leq 1.0$ using 18 calculated parcels of the Taylor series, and the mentioned parcels for boundary conditions at $z^{*}=0$. The values of the control parameters $A_{1}$ and $\Phi_{1}$ are shown in Fig. 3, while $S$ was taken as $S=1000$. Experimental data of [9] are also shown for qualitative comparisons. To allow this comparison, the $z$ axis is normalized with the usual boundary layer thickness $\delta$ at $n=0.01$. The positions $z^{*}$ at which $\delta$ is attained are shown in Table 3. It must be commented that the data of [9] produce $S \sim 1.0$, while the calculations were performed with $S=1000$ to facilitate the convergence of the Taylor series (reducing the number of parcels of the series). The present comparison is, thus, qualitative. It is firstly observed that the calculated $n$ profile follows the general trend of experimental observations of the literature, i.e., decreases from its maximum value ( $n=1.0$ at the interface) to the value $n=0.01$ at $\mathrm{z} / \delta=1.0$. It is further observed that, for $\Phi_{1}<10^{3}$, the different curves converge to the condition represented by the thin gray lines. Table 3 also shows that $\delta^{*}$ converges to $\sim 0.2165$ for the lower values of $\Phi_{1}$.

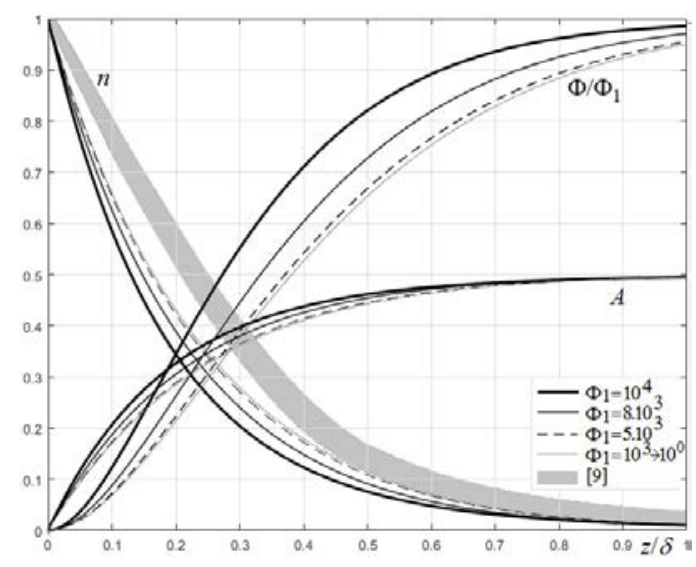

Figure 3: Profiles of variables related to mass transfer in the boundary layer: $n, A$ and $\Phi / \Phi_{1}$. Parameter values: $S=1000, A_{1}=0.5$ and $\Phi_{1}$ shown in the figure. The gray cloud represents the data of [9]. 
Table 3: Control parameters $A_{1}, \Phi_{1}$ and characteristic lengths $\delta, \delta_{c}$ of the boundary layer.

\begin{tabular}{|c|c|c|c|c|c|c|}
\cline { 2 - 7 } \multicolumn{1}{c|}{} & \multicolumn{2}{c|}{$A_{1}=0.45$} & \multicolumn{2}{c|}{$A_{1}=0.50$} & \multicolumn{2}{c|}{$A_{1}=0.75$} \\
\hline$\Phi_{1}$ & $\begin{array}{c}\delta^{*}=\delta / E \\
(n=0.01)\end{array}$ & $\begin{array}{c}\delta^{*}{ }_{c}=\delta_{c^{\prime}} / E \\
(n=0.01)\end{array}$ & $\begin{array}{c}\delta^{*}=\delta / E \\
(n=0.01)\end{array}$ & $\begin{array}{c}\delta^{*}{ }_{c^{\prime}}=\delta_{c^{\prime}} / E \\
(n=0.01)\end{array}$ & $\begin{array}{c}\delta^{*}=\delta / E \\
(n=0.01)\end{array}$ & $\begin{array}{c}\delta^{*}{ }_{c}=\delta_{c^{\prime}} / E \\
(n=0.01)\end{array}$ \\
\hline $1.010^{4}$ & 0.4010 & 0.1386 & 0.3145 & 0.08314 & 0.2190 & 0.07390 \\
\hline $8.010^{3}$ & 0.3058 & 0.08314 & 0.2513 & 0.07390 & 0.2181 & 0.07390 \\
\hline $5.010^{3}$ & 0.2284 & 0.07390 & 0.2229 & 0.07390 & 0.2173 & 0.07390 \\
\hline $1.010^{3}$ & 0.2169 & 0.07390 & 0.2169 & 0.07390 & 0.2166 & 0.07390 \\
\hline $1.010^{2}$ & 0.2170 & 0.07390 & 0.2165 & 0.07390 & 0.2165 & 0.07390 \\
\hline $1.010^{1}$ & 0.2165 & 0.07390 & 0.2165 & 0.07390 & 0.2165 & 0.07390 \\
\hline $1.010^{0}$ & 0.2165 & 0.07390 & 0.2165 & 0.07390 & 0.2165 & 0.07390 \\
\hline
\end{tabular}

In Fig. 3, $\Phi / \Phi_{1}$ follows the expected behaviour of increasing turbulent mass flux with $z / \delta$ in the boundary layer. In real situations for fixed liquid volumes, the profile of $\Phi / \Phi_{1}$ firstly increases near the interface (in the boundary layer), and further decreases tending to zero in the bulk liquid (outside the boundary layer), as already discussed by [11].

From Fig. 3, $A$ also increases with $z / \delta$ in the boundary layer, following experimental data obtained by [9] and shown in [16]. This result extends the discussion of [27], in which the focus was the stationary solution of RSW for constant $A$, showing now a wider range of application. $A=0.0$ is characteristic for damping (attenuation) of fluctuations due to molecular diffusivity near the interface. Because molecular diffusion is important in the so called "outer diffusive layer" (see the BOKVS interfacial structure in [22] and [27], for example), low values of $A$ also occur in this layer.

In Table $3, \delta^{*}$, is the position of the peak at $z^{*}$ of the function that expresses the RMS fluctuation of concentration, and used in the subsequent discussion.

Fig. 4 shows the same curves of Fig. 3 , but for $A_{1}=0.75$. It can be seen that $n, A$, and $\Phi$ collapse to single curves. Because $A$ is a measure of attenuation due to diffusivity, this result suggests that lower attenuations generate profiles less sensitive (or no sensitive) to variations of $\Phi_{1}$, the turbulent mass flux taken as control parameter, for $1.0 \leq \Phi_{1} \leq 10^{4}$ and $S=1000$.

Fig. 5(a) shows the comparison between measured and calculated concentration fluctuation intensities, given as the root mean square $c^{\prime}$ of the concentration fluctuations $c$, $c^{\prime}=\sqrt{\overline{c^{2}}}$, and the RSW equation $c^{\prime}=A \sqrt{n(1-n)}$ (see [16]). The experimental data were obtained by [9] and discussed by [16] and [27]. The $z$ axis is normalized with the position of the peak of $c^{\prime}$ in order to facilitate comparisons with measurements. As can be seen, the calculated profile of $A$ follows the cloud of measured profiles.

Having the position of the peak of $c^{\prime}$ of Fig. 5(a), it was also possible to compare experimental and theoretical profiles of $A$ against $z / \delta_{c}$, as shown in Fig. 5(b). The growing of $A$ with $z / \delta_{c}$, for $z / \delta_{c} \rightarrow 0.0$ is observed in both the measured data and the theoretical curve, and they show similar slopes for the interval $0.0<\mathrm{z} / \delta_{c},<\sim 1.3$. As differences between the two results it is observed that the cloud of data decreases for $z / \delta_{c},>\sim 2.5$, which is not followed by the calculated curve. This last theoretical result is more closely related to the boundary conditions applied at $z^{*}=1$, and to the imposed value $S=1000$ for the momentary convergence analysis, which not necessarily reproduce the data of [9]. 


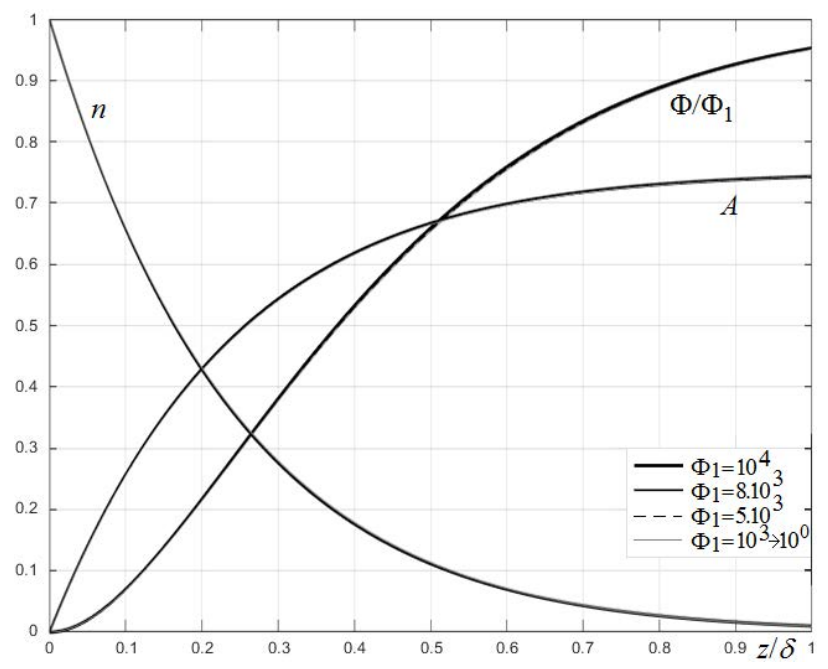

Figure 4: Profiles of variables related to mass transfer in the boundary layer: $n, A$ and $\Phi / \Phi_{1}$. Parameter values: $S=1000, A_{1}=0.75$ and $\Phi_{1}$ shown in the figure. The evolution of the different variables collapse to single curves.

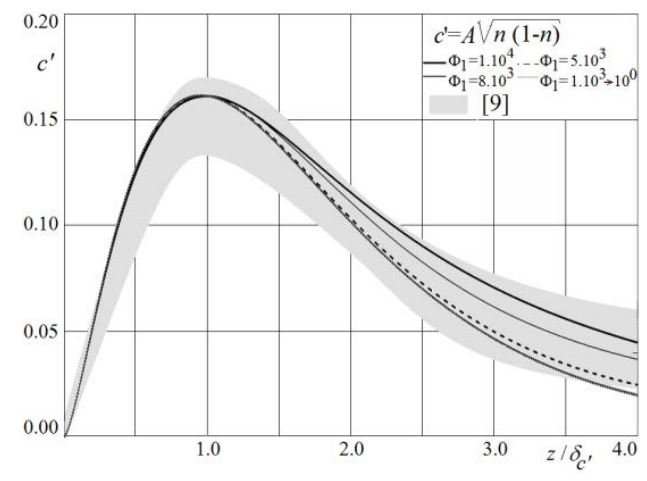

(a)

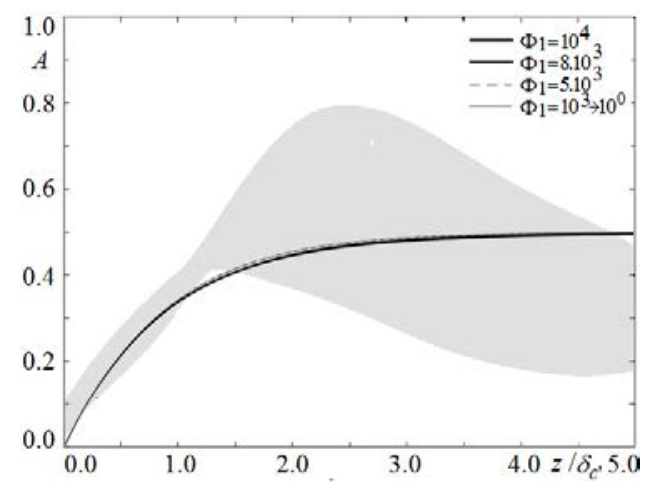

(b)

Figure 5: The comparison between measured and calculated concentration fluctuation intensities: (a) Profiles of the experimental $c^{\prime}=\sqrt{\overline{c^{2}}}$ and the theoretical RSW equation $c^{\prime}=A \sqrt{n(1-n)}$ for $A_{1}=0.5$; (b) Profiles of experimental and theoretical $A$. The solid line is the calculated curve for $A_{1}=0.5$. The gray cloud represents data of [9].

In the present study $\Phi / \Phi_{1}$ increases with the second power of $z^{*}$ for $z^{*} \rightarrow 0.0$, one of the possibilities discussed by [11]. This result shows that the proposed Taylor series of $\Phi$ converges, and that the condition $d \Phi / d z^{*}=0.0$ at $z^{*}=0.0$ is adequate to obtain realistic trends. The proportionality between $\Phi / \Phi_{1}$ and $z^{* 2}$ for free slip surfaces may thus be incorporated by the present formulation. This result is shown in Fig. 6, where the theoretical results of $\Phi / \Phi_{1}$ obtained for $A_{1}=0.5$ are plotted against $z^{*}$ in logarithm scale. 


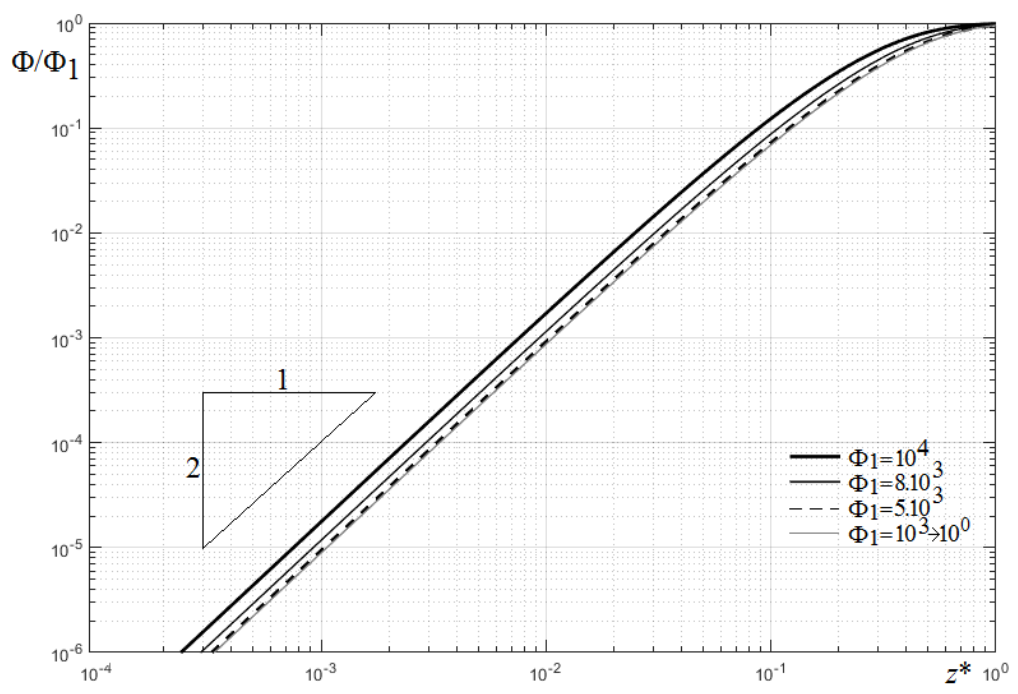

Figure 6: Calculated profile of $\Phi / \Phi_{1}$. The trend to the slope 2.0 close to the interface shows the adequacy of the result, considering discussions and experimental data of the literature (see [11]), and the convergence of the method. $A_{1}=0.5$.

The results presented in Figs 3-6 express the evolution of relevant variables in concentration boundary layers. It is shown that the RWS tool is adequate to quantify scalar boundary layers, being dependent of a number of control parameters $\left(A_{1}, \Phi_{1}\right.$, and $\left.S\right)$. These parameters allow quantifying the whole set of coefficients of the Taylor series that expresses $n$, (partition function) $A$ (complementary of the reduction function), and $\Phi$ (nondimensional turbulent mass flux). In the present study 18 coefficients were calculated for each series. By reducing the value of $S$, more parcels should be added to the solution.

\section{CONCLUSIONS}

The mathematical tool of Random Square Waves (RSW) for the study of gas exchanges across a turbulent gas-liquid interface was described. It was shown that the originally open set of statistical equations for turbulence can be closed by applying this tool. The condition of transient one dimensional mass transfer considering a variable reduction function $\alpha_{f}$ was adopted for the calculations. It was shown that the RSW tool for $1 \mathrm{D}$ transfer depends on three functions: the partition function $n$, the reduction function $a_{f}$ (or its complementary $A=1$ $a_{f}$ ), and the turbulent flux $\Phi$. As a consequence, only three equations are needed to calculate the turbulent parameters in the boundary layer.

The calculations show the first results of a novel form of quantifying the turbulent parameters $n, A$, and $\Phi$ in the concentration boundary layer, for which Taylor series are used.

To obtain the successive derivatives of the variables under study that are needed for the Taylor series, adequate boundary conditions for the normalized concentration profile $n$ were imposed at the normalized position $z^{*}=1.0$, in the form: $n^{*}\left(z^{*}=1.0\right)=0.0$ and $\mathrm{d} n / \mathrm{d} z^{*}\left(z^{*}=1.0\right)=0.0$. Because the derivatives involve negative powers of the parameter $S$ of the RSW tool, the present first calculations were performed using $S=1000$, so that only 18 parcels were used to quantify the coefficients of the Taylor series of $n, A$, and $\Phi$. To consider boundary conditions at $z^{*}=0$, one parcel was added to the series of $n$ and $A$ and two parcels were added to the series of $\Phi$. All derivatives depend on the values of $A$ and $\Phi$ at $z^{*}=1.0$, 
expressed as $A_{1}$ and $\Phi_{1}$, respectively. In the present study three values of $A_{1}$ were tested, together with seven values of $\Phi_{1}$, as shown in Table 3 .

It was observed that the Taylor series applied to the RSW tool furnished good results:

1. The calculated profile of the normalized concentration, $n$ (or the partition function), showed the general behaviour observed in experimental results of the literature, decreasing from the value $n=1.0$ at the interface to the value $n=0.01$ at the end of the boundary layer.

2. The calculated profile of the intensity of the concentration fluctuations c' superposed very well measured profiles.

3. The profiles of the functions $A$ increased with the distance to the interface, following experimental results found in the literature. Close to the interface $\left(z^{*}=0\right)$ the calculated evolution of $A$ superposed well measured evolutions of the literature. Differences observed between theoretical results and experimental data for great distances to the interface may be related to the boundary conditions adopted for $z^{*}=1.0$. This situation indicates possible future steps in this research line.

4. The calculated profiles of the functions $\Phi$ are adequate. In the present calculations the turbulent mass flux $\Phi$ increased with the second power of the distance to the interface, a possibility that is discussed in the literature.

5. The RSW tool showed to be adequate for boundary layer studies.

Having obtained the above described positive results of the first application of Taylor series to the RSW tool, the reproduction of real conditions will be now pursued related to the value of $S$ and alternative boundary conditions for $z^{*}=1.0$. It implies in lowering the value of $S$, which results in using a larger number of terms in the Taylor series. Both measures are necessary and can now be conducted in the light of the present positive results.

\section{ACKNOWLEDGEMENTS}

The authors gratefully acknowledge CNPq (National Council for Scientific and Technological Development, Brazil) for the financial support. The first author thanks CAPES for the fellowship PPG-AEM-EESC-USP. The second author mentions the process CNPq 307105/2015-6 that assures the continuity of the research line in statistical turbulence and RSW.

\section{REFERENCES}

[1] Gulliver, J.S., (eds), Introduction to Chemical Transport in the Environment, Cambridge University Press: New York, 2007.

[2] Jorgensen, S.E. \& Gromiec, M.J., Chapter 3: Reaeration. Developments in Environmental Modelling, Elsevier: Amsterdam, vol. 14, pp. 33-64, 1989.

[3] Bennett, J.P. \& Rathbun, R.E., Reaeration in Open-Channel Flow, Washington, DC: US Government Printing Office, 1972.

[4] Janzen, J.G., Jirka, G.H. \& Schulz, H.E., Details of air-water gas transfer: experiments with precision - in Portuguese. Proceedings of the 16th Brazilian Conference on Water Resources (XVI Simpósio Brasileiro de Recursos Hídricos), pp. 1-17, 2005.

[5] Janzen, J.G., Schulz, H.E. \& Jirka, G.H., Details of air-water gas transfer - in Portuguese. Brazilian Journal of Water Resources, 11(4), pp. 153-161, 2006.

[6] Chu, C.R. \& Jirka, G.H., Turbulent gas flux measurements below the air-water interface of a grid-stirred tank. International Journal of Heat and Mass Transfer, 35(8), pp. 1957-1968, 1992. 
[7] Herlina, Gas Transfer at the Air-Water Interface in a Turbulent Flow Environment, Dissertation series, Karlsruhe: Institute for Hydromechanics of the University of Karlsruhe, 2005.

[8] Herlina \& Jirka, G.H., Application of LIF to investigate gas transfer near the air-water interface in a grid-stirred tank. Experiments in Fluids, 37(3), 341-349, 2004.

[9] Janzen, J.G., Gas Transfer Near the Air-Water Interface in Oscillating-Grid Tanks and Proprieties of Isotropic Turbulent Flows (in Portuguese), Dissertation, São Paulo: São Carlos School of Engineering at the University of São Paulo, 2006.

[10] Janzen, J.G., Herlina, H., Jirka, G.H., Schulz, H.E. \& Gulliver, J.S., Estimation of mass transfer velocity based on measured turbulence parameters. American Institute of Chemical Engineers Journal, 56(8), pp. 2005-2017, 2010.

[11] Janzen, J.G., Schulz, H.E. \& Jirka, G.H., Turbulent gas flux measurements near the air-water interface in an oscillating-grid tank. Proceedings of the 2010 Gas Transfer at Water Surfaces Conference, pp. 65-77, 2011.

[12] Herzog, A.G., Imaging of Water-Sided Gas-Concentration Fields at a Wind-Driven, Wavy Air-Water Interface, Dissertation, Heidelberg: Faculties for the Natural Sciences and for Mathematics Institute of the Ruperto-Carola University of Heidelberg, 2010.

[13] Friedl, F., Investigating the Transfer of Oxygen at the Wavy Air-Water Interface Under Wind-Induced Turbulence, Dissertation, Heidelberg: Faculties for the Natural Sciences and for Mathematics Institute of the Ruperto-Carola University of Heidelberg, 2013.

[14] Krah, N., Visualization of Air and Water-Sided Concentration Profiles in Laboratory Gas Exchange Experiments, Dissertation, Heidelberg: Faculties for the Natural Sciences and for Mathematics Institute of the Ruperto-Carola University of Heidelberg, 2014.

[15] Schulz, H.E., Lopes Jr., G.B., Simões, A.L.A. \& Lobosco, R.J., (eds), Chapter 1: Onedimensional turbulent transfer using random square waves - scalar/velocity and velocity/velocity interactions. Hydrodynamics: Advanced Topics, Rijeka: InTech, pp. 3-34, 2011.

[16] Schulz, H.E. \& Janzen, J.G., Concentration fields near air-water interfaces during interfacial mass transport: oxygen transport and random square wave analysis. Brazilian Journal of Chemical Engineering, 26(3), pp. 527-536, 2009.

[17] Lacassagne, T., El-Hajem, M., Morge, F., Simoens, S. \& Champagne, J.Y., Study of gas liquid mass transfer in a grid stirred tank. Oil \& Gas Science and TechnologyRevue d'IFP Energies nouvelles, 72(1), p. 7, 2017.

[18] Tamburrino, A. \& Martínez, N., Wave and wind effects on the oxygen transfer across an air-water interface: An experimental study. The Canadian Journal of Chemical Engineering, 95(8), pp. 1594-1604, 2017.

[19] Herlina, H. \& Wissink, J.G., Direct numerical simulation of turbulent scalar transport across a flat surface. Journal of Fluid Mechanics, 744, pp. 217-249, 2014.

[20] Herlina, H. \& Wissink, J.G., Simulation of air-water interfacial mass transfer driven by high-intensity isotropic turbulence. Journal of Fluid Mechanics, 860, pp. 419-440, 2019.

[21] McCready, M.J., Vassiliadou, E. \& Hanratty, T.J., Computer simulation of turbulent mass transfer at a mobile interface. AIChE Journal, 32(7), pp. 1108-1115, 1986.

[22] Brumley, B.H. \& Jirka, G.H., Near-surface turbulence in a grid-stirred tank. Journal of Fluid Mechanics, 183, 235-263, 1987.

[23] Sirkar, K.K. \& Hanratty, T.J., Relation of turbulent mass transfer to a wall at high Schmidt numbers to the velocity field. Journal of Fluid Mechanics, 44(3), pp. 589603, 1970. 
88 Computational and Experimental Methods in Multiphase and Complex Flow X

[24] Schulz, H.E. \& Schulz, S.A.G., Modelling below-surface characteristics in water reaeration. Proceedings: Water Pollution: Modelling, Measuring and Prediction, pp. 441-454, 1991.

[25] Schulz, H.E., Simões, A.L. \& Janzen, J.G., Statistical approximations in gas-liquid mass transfer. Proceedings of the 2010 Gas Transfer at Water Surfaces Conference, pp. 208-221, 2011.

[26] Schulz, H.E. \& Gonçalves, B.B., Solutions of scalar mean profiles close to gas-liquid interfaces under turbulent free slip motion. Proceedings of the Eight Conference on Computational Methods in Multiphase Flow, pp. 33-44, 2015.

[27] Schulz, H.E., Lavín, F.A.L. \& Gonçalves, B.B., Turbulence aspects of mass transfer in the thin interfacial region of the concentration boundary layer in gas-liquid systems. International Journal of Computational Methods and Experimental Measurements, pp. 6(1), pp. 186-197, 2018. 